\title{
Analysis of Exonic Mutations Leading to Exon Skipping in Patients with Pyruvate Dehydrogenase E1 $\alpha$ Deficiency
}

\author{
ALESSANDRA KUPPER CARDOZO, LINDA DE MEIRLEIR, INGE LIEBAERS, AND \\ WILLY LISSENS \\ Center for Medical Genetics [A.K.C., L.D.M., I.L., W.L.] and Pediatric Neurology [L.D.M.], University \\ Hospital, Vrije Universiteit Brussel, 1090 Brussels, Belgium.
}

\begin{abstract}
The pyruvate dehydrogenase $(\mathrm{PDH})$ complex is situated at a key position in energy metabolism and is responsible for the conversion of pyruvate to acetyl CoA. In the literature, two unrelated patients with a PDH complex deficiency and splicing out of exon 6 of the PDH E1 $\alpha$ gene have been described, although intronic/exonic boundaries on either side of exon 6 were completely normal. Analysis of exon 6 in genomic DNA of these patients revealed two exonic mutations, a silent and a missense mutation. Although not experimentally demonstrated, the authors in both publications suggested that the exonic mutations were responsible for the exon skipping. In this work, we were able to demonstrate, by performing splicing experiments, that the two exonic mutations described in the PDH E1 $\alpha$ gene lead to aberrant splicing. We observed a disruption of the predicted wild-type pre-mRNA secondary structure of exon 6 by the mutated sequences described. However, when we constructed
\end{abstract}

ABSTRACT

mutations that either reverted or disrupted the wild-type predicted pre-mRNA secondary structure of exon 6 , we were unable to establish a correlation between the aberrant splicing and disruption of the predicted structure. The mutagenic experiments described here and the silent mutation found in one of the patients suggest the presence of an exonic splicing enhancer in the middle region of exon 6 of the PDH E1 $\alpha$ gene. (Pediatr Res 48: 748-753, 2000)
ESE, exonic splicing enhancer
mfe, minimum free energy
PDH, pyruvate dehydrogenase
RT-PCR, reverse transcriptase PCR
SR, serine-arginine

\section{Abbreviations}

PDH complex deficiency is one of the most common defined genetic defects of mitochondrial energy metabolism (1). It is a severe disease, which results in early death in the majority of affected patients (2). Most of the cases are sporadic and result from a new mutation arising within the germ cells of one of the parents. However, familial cases have also been described (3-5).

The majority of the molecular defects of the PDH complex have been localized in the $\mathrm{E} 1 \alpha$ subunit gene at chromosome Xp22.1 [gene symbol PDHA1; MIM\# 312170; (6)]. More than 120 patients with PDH E1 $\alpha$ deficiency have been described, and at least 75 different mutations in the coding region have been reported (7). Two cases of splicing out of exon 6 from the PDH E1 $\alpha$ mRNA have been found $(8,9)$. De Meirleir et al. (8), studying a family with Leigh's encephalomyelopathy, found a

Received January 10, 2000; accepted July 22, 2000.

Correspondence and reprint requests: Willy Lissens, Ph.D., Center for Medical Genetics, University Hospital VUB, Laarbeeklaan 101, 1090 Brussels, Belgium.

Supported by the National Fund for Scientific Research (NFWO) grant 3.0065.87, the Fund for Scientific Research Flanders (FWO Vlaanderen) grant G0181.98, and the Research Council of the Vrije Universiteit Brussel (VUB), Brussels, Belgium. partial skipping of the entire exon 6 in two affected boys. Analysis of the genomic DNA of these individuals revealed a silent mutation at nucleotide 45 of exon 6 [cDNA position 660 according to Lissens et al. (7)]. Chun et al. (9) found a deletion of exon 6 in all of the cDNAs examined in a female patient who died of severe neonatal lactic acidosis. At the genomic level, this patient was heterozygous for a missense mutation at nucleotide 13 of exon 6 (cDNA position 628). Although not experimentally demonstrated, the authors of both studies suggested that the exonic mutations were responsible in some way for the transcript with the missing exon.

It is estimated that up to $15 \%$ of all point mutations causing human genetic disorders do so by resulting in aberrant mRNA splicing. The majority of these mutations are located in the consensus splice sites at the intron/exon borders (10). However, a small proportion of these mutations affect exon sequences that are distinct from the consensus sites $(8,9,11-20)$, but it is not clear how these exonic mutations affect splicing. It was suggested $(8,11,18)$ that the exonic mutations may lead to aberrant splicing owing to a disruption of the pre-mRNA secondary structure. Although the exact role of the secondary 
Zaventem, Belgium). The conditions were $95^{\circ} \mathrm{C}$ for $12 \mathrm{~min}$ and 20 cycles of $94^{\circ} \mathrm{C}$ for $45 \mathrm{~s}, 59^{\circ} \mathrm{C}$ for $45 \mathrm{~s}$, and $72^{\circ} \mathrm{C}$ for $1 \mathrm{~min}$, with a final extension of $10 \mathrm{~min}$ at $72^{\circ} \mathrm{C}$. The products were visualized on $2.5 \%$ agarose gels stained with ethidium bromide; part of the products were used for sequencing. When a PCR reaction resulted in two fragments, these fragments were separated on a $15 \%$ polyacrylamide gel and cut out to be sequenced.

To determine the optimal conditions, PCRs were run for 16, $18,20,22,24,26$, and 28 cycles. Using 20 cycles, the PCR was still in the logarithmic phase. All the RT-PCRs were performed three times using RNA from different transfections.

Calculation of the mfe pre-mRNA secondary structures. The mfe pre-mRNA secondary structures were calculated using the program RNAdraw V1.1 (30). The calculations were imported from the RNA fold program included in the Vienna RNA package (31).

Site-directed mutagenesis. Site-directed mutagenesis was performed using the Quick Change Site-Directed Mutagenesis Kit (Stratagene, La Jolla, CA, U.S.A.). The double-stranded vector containing wild-type exon 6 of the PDH E1 $\alpha$ gene was used as a template for all mutageneses with an exception for mutation $708+118 \mathrm{~T} \rightarrow \mathrm{C}$, for which the plasmid containing mutation $628 \mathrm{G} \rightarrow \mathrm{A}$ was used as a template. Primers used are listed in Table 1. Positions of mutations in exon 6 are shown in Fig. 1. The results of all the mutagenesis studies were confirmed by sequence analysis.

\section{RESULTS}

Cloning and expression of normal and mutant exon 6 E1 $\alpha$ sequences. To verify whether the exonic mutation found in patient G.D. [660A $\rightarrow \mathrm{G},(8)]$ was responsible for the splicing out of exon 6, we used the exon trap approach. Plasmids containing wild-type or mutant $660 \mathrm{~A} \rightarrow \mathrm{G}$ exon 6 were transfected in COS-7 cells. RT-PCR from total RNA was performed and resulted in almost equal quantities of amplification products of $339 \mathrm{bp}$ and $246 \mathrm{bp}$ for the wild-type (Fig. 2A, lane 3), and a product of $246 \mathrm{bp}$ and a barely detectable product of 339 bp for mutant $660 \mathrm{~A} \rightarrow \mathrm{G}$ (Fig. 2A, lane 4). Sequencing revealed that the product of 339 bp contained the PDH E1 $\alpha$ exon 6 plus insulin exonic sequences (derived from the vector) and that the product of $246 \mathrm{bp}$ contained only the insulin exonic sequences without exon 6.

The other exonic mutation leading to aberrant splicing of exon 6 of the PDH E1 $\alpha$ gene was described in patient 2641 from the study by Chun et al. (9). To verify whether this mutation $628 \mathrm{G} \rightarrow \mathrm{A}$ ( $\mathrm{G}$ to A substitution at nucleotide 13 of exon 6) was also responsible for the aberrant splicing, a site-directed mutagenesis in exon 6 from the wild-type PDH E1 $\alpha$ gene was performed, mimicking the described mutation. After transfections in COS-7 cells, we observed complete splicing out of exon 6 for this sequence (Fig. 2B, lane 5).

An updated computer program, RNAdraw V1.1 (30), was used to predict the mfe secondary structures of the $93 \mathrm{bp}$ from exon 6 in wild-type, mutant $660 \mathrm{~A} \rightarrow \mathrm{G}$, and mutant $628 \mathrm{G} \rightarrow \mathrm{A}$ (data not shown). By comparison of the predicted structures, it was observed that both mutations disrupt the wild-type conformation.

Site-directed mutagenesis based on predicted mfe pre$m \boldsymbol{R} N A$ secondary structures. Because the exonic mutations in the PDH E1 $\alpha$ gene affect splicing and disrupt the predicted wild-type secondary structure, we decided to test whether there is a relationship between the disruption of the predicted secondary structure and the splicing out of exon 6. For this purpose, site-directed mutagenesis in this exon was performed based on the RNAdraw program.

The first mutagenesis was designed in a way such that disruption of the mfe secondary structure of exon 6 was obtained without changing the amino acid glycine (G195). It was a $\mathrm{C}$ to $\mathrm{A}$ substitution at nucleotide 75 of exon 6 $(690 \mathrm{C} \rightarrow \mathrm{A})$. Transient transfection of the mutated construct in COS-7 cells showed, by RT-PCR, two amplification products of $339 \mathrm{bp}$ and $246 \mathrm{bp}$ (Fig. 2B, lane 4). The ratio between the intensity of these products was similar to that found in the wild-type construct.

When predicting the secondary structures above, we followed the same procedure as Chun et al. (9), Steinsgrimsdottir et al. (11), and Fukao et al. (12). These authors took into consideration only the exonic sequences for predicting the mfe pre-mRNA structures, and interactions between exonic/

Table 1. Primers used for mutagenesis

\begin{tabular}{|c|c|}
\hline Mutation & Primer* \\
\hline $628 \mathrm{G} \rightarrow \mathrm{A}$ & 1. 5'-GGTAGGTGCCCCTGGGCACTGGGATTGCTCTAGCC-3' \\
\hline (patient 2641) & 2. 5'-GGCTAGAGCAATCCCAGT̄GCCCAGGGGCAGCTAAC-3' \\
\hline $690 \mathrm{C} \rightarrow \mathrm{A}$ & 1. 5'-CTGGTTAGCAGCACCATCTCCATATAAAGTCAGGCAG-3' \\
\hline \multirow[t]{2}{*}{$666 \mathrm{~T} \rightarrow \mathrm{C}$} & 1. 5'-CCTGTAAGTATAATGGAAAAGACGAGGTCTGCCTGACTTTATATG-3' \\
\hline & 2. 5'-CATATAAAGTCAGGCAGACCTCGTCTTTTCCATTATACTTACAGG-3' \\
\hline $671 \mathrm{~T} \rightarrow \mathrm{C}$ & 1. 5'-GTATAATGGAAAAGATGAGGCCT-GCCTGACTTTATATGGCG-3' \\
\hline $708+118 \mathrm{~T} \rightarrow \mathrm{C}$ & 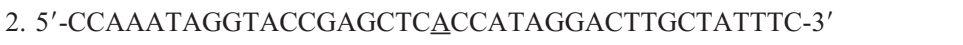 \\
\hline \multirow[t]{2}{*}{$661 \mathrm{~A} \rightarrow \mathrm{C}$} & 1. 5'-CCTGTAAGTATAATGGACAAAGATGAGGTCTGCCTG-3' \\
\hline & 2. 5'-CAGGCAGACCTCATCTTḠTCCATTATACTTACAGG-3' \\
\hline \multirow[t]{2}{*}{ 640delAA } & 1. 5'-CTGTAAGTATAATGG_AAGATGAGGTCTGCCTGAC-3' \\
\hline & 2. 5'-GTCAGGCAGACCTCATCTT__CCATTATACTTACAG-3' \\
\hline
\end{tabular}

* The underlined nucleotides correspond to the mutated or deleted ones. 


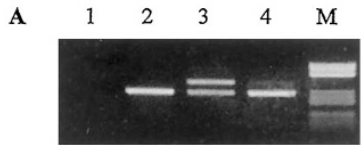

C

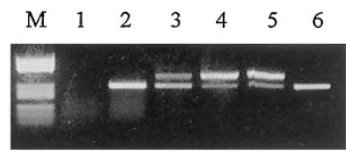

B

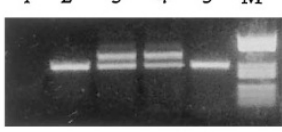

D

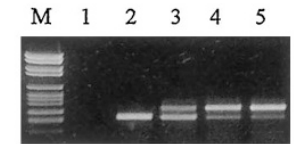

Figure 2. RT-PCR analysis of the PDH E1 $\alpha$ gene exon 6. Total RNA was isolated from transiently transfected COS-7 cells and used for RT-PCR analysis. Representative results for triplicate experiments are shown. In all the figures, lanes 1 to 3 correspond to mock-transfected cells, cells transfected with empty vector and wild-type, respectively. A, lane 4 , mutant $660 \mathrm{~A} \rightarrow \mathrm{G}$. The wild-type constuct (lane 3) shows two bands: the upper band (339 bp) contains exon 6, whereas in the lower band ( $246 \mathrm{bp})$, exon 6 is lost. In the mutant sequence of patient G. D. (lane 4), we observed mainly the 246-bp band, indicating that most of the mRNA has lost exon 6. B, lanes 4 and 5 , mutant $690 \mathrm{C} \rightarrow \mathrm{A}$ and mutant $628 \mathrm{G} \rightarrow \mathrm{A}$, respectively. Mutant $628 \mathrm{G} \rightarrow \mathrm{A}$ (lane 5) contains only the band of $246 \mathrm{bp}$, indicating that all of the mRNA skipps exon 6. Mutant $690 \mathrm{C} \rightarrow \mathrm{A}$ (lane 4 ) maintains the same pattern as wild-type. $C$, lanes 4-6, mutants $666 \mathrm{~T} \rightarrow \mathrm{C}, 671 \mathrm{~T} \rightarrow \mathrm{C}$ and $708+118 \mathrm{~T} \rightarrow \mathrm{C}$, respectively. Mutants $666 \mathrm{~T} \rightarrow \mathrm{C}$ and $671 \mathrm{~T} \rightarrow \mathrm{C}$ (lanes 4 and 5 , respectively) show the same pattern of splicing as wild-type. The ratio between the intensities of the fragments retaining (339 bp) and having lost exon 6 (246 bp) were higher in these mutants than in the wild-type. Mutant $708+118 \mathrm{~T} \rightarrow \mathrm{C}$ (lane 6) had $100 \%$ skipping of exon 6. D, lanes 4 and 5 , mutants $661 \mathrm{~A} \rightarrow \mathrm{C}$ and 660delAA, respectively. Both mutants show the same pattern of splicing as wild-type, but the ratio between the intensity of the fragments retaining and skipping exon 6 is higher in the mutants than in the wild-type. Markers $(M)$ for $A, B$, and $C, 64$ to $587 \mathrm{bp}$; marker for $D, 124$ to 2176 bp (Boehringer Mannheim, Brussels, Belgium).

intronic sequences in the folding of the pre-mRNA were not considered. Again using the RNA draw program, we redrew the structures of wild-type and mutant $(660 \mathrm{~A} \rightarrow \mathrm{G}, 628 \mathrm{G} \rightarrow \mathrm{A}$, and $690 \mathrm{C} \rightarrow \mathrm{A}$ ) exon 6 of the $\mathrm{PDH} \mathrm{E} 1 \alpha$ gene, but this time taking into consideration part of the surrounding introns (Fig. $3, A-D)$. We observed that at least $150 \mathrm{bp}$ of each intron had to be included in the calculations to obtain a stable structure.

To test the hypothesis that the predicted mfe secondary structure of exon 6 influences the splicing process, we designed three new mutations, based on calculated structures including intronic sequences: 1 ) a $\mathrm{T} \rightarrow \mathrm{C}$ substitution at nucleotide 51 of exon 6 (mutation 666T $\rightarrow$ C); this mutation does not change the amino acid asparagine (D187) but the wild-type pre-mRNA secondary structure is disrupted (Fig. $3 E$ ); 2) a $\mathrm{T} \rightarrow \mathrm{C}$ substitution at nucleotide 56 of exon 6 (mutation $671 \mathrm{~T} \rightarrow \mathrm{C}$ ). The amino acid valine is changed into an alanine (V189A), and the pre-mRNA secondary structure is the same as for the wildtype; 3) a mutation that does not affect the consensus splice sequences was made in intron 6 ( $\mathrm{T}$ to $\mathrm{C}$ substitution $118 \mathrm{bp}$ downstream of the $5^{\prime}$ splice site of intron 6 mutation $708+118 \mathrm{~T} \rightarrow \mathrm{C})$ in the construct containing mutation $628 \mathrm{G} \rightarrow \mathrm{A}$. This mutation was designed in a way such that the disrupted secondary structure of exon 6 containing mutation $628 \mathrm{G} \rightarrow \mathrm{A}$ was reverted to the wild-type.

After mutagenesis and expression in COS-7 cells, we observed that the mutation $671 \mathrm{~T} \rightarrow \mathrm{C}$, with a conserved secondary structure, showed RT-PCR fragments of $339 \mathrm{bp}$ and $246 \mathrm{bp}$ (Fig. 2C, lane 5). The ratio between the intensity of fragments retaining and of those skipping exon 6 was higher in this

A

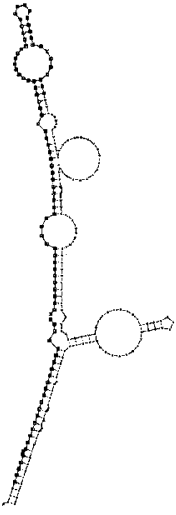

D

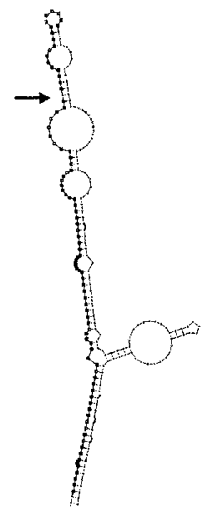

B

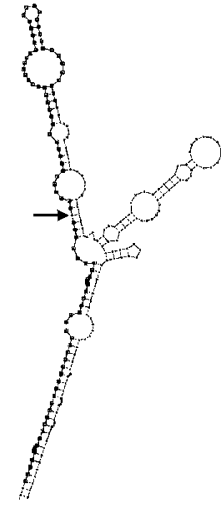

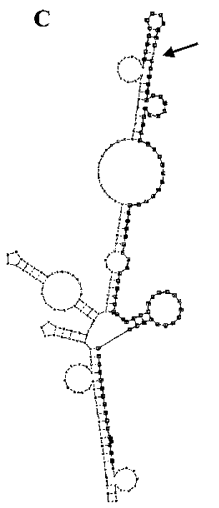

$\mathbf{E}$

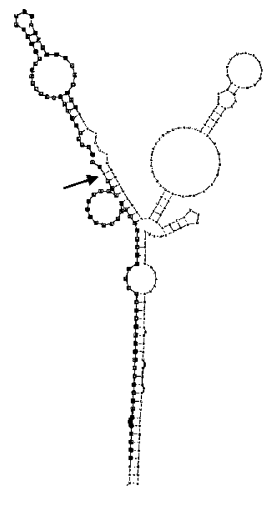

Figure 3. View of part of the predicted pre-mRNA mfe secondary structure of exon 6 and part of the boundary introns of the PDH E1 $\alpha$ gene. $A$, wild-type. $B$, mutation $660 \mathrm{G} \rightarrow \mathrm{A}$ (patient G. D). $C$, mutation $628 \mathrm{~A} \rightarrow \mathrm{G}$ (patient 2641). $D$, mutation $690 \mathrm{C} \rightarrow$ A. $E$, mutation $666 \mathrm{~T} \rightarrow$ C. The exonic sequence is in bold, and the arrows indicate the mutated bases.

construct when compared with the wild-type. However, the construct containing mutation $666 \mathrm{~T} \rightarrow \mathrm{C}$, with a disrupted secondary structure, also showed these two fragments and in the same proportions as mutation $671 \mathrm{~T} \rightarrow \mathrm{C}$ (Fig. 2C, lane 4). The double construct with mutation $708+118 \mathrm{~T} \rightarrow \mathrm{C}$ showed $100 \%$ skipping of exon 6 (Fig. 2C, lane 6).

Mutation in a purine-rich region of exon 6 of the PDH E1 $\alpha$ gene. The mutation of patient G.D. is located in a purine-rich region that could be acting as an ESE (Fig. 1). We decided to test whether this is a conserved region for the recognition of exon 6 by the spliceosome complex. For this reason, we constructed two new mutations: 1 ) an $\mathrm{A} \rightarrow \mathrm{C}$ substitution at nucleotide 46 of exon 6 (mutation $661 \mathrm{~A} \rightarrow \mathrm{C}$ ); the amino acid lysine is changed to glutamine (K186Q); and 2) a deletion was made to remove two of the four consecutive adenines of the purine-rich region (mutation 660delAA).

After transfection in COS-7 cells, we observed RT-PCR fragments of $339 \mathrm{bp}$ and $246 \mathrm{bp}$ for both mutations (Fig. 2D, lanes 4 and 5). The ratio between the intensity of the fragments retaining and of those skipping exon 6 was higher in these constructs when compared with the wild-type.

\section{DISCUSSION}

In the present study, we demonstrate by using exon trap analysis that the two exonic mutations described in exon 6 of 
the PDH E1 $\alpha$ gene $(8,9)$ indeed cause aberrant splicing of this exon. Several cases of exonic mutations presumed to lead to aberrant splicing have been described in the literature, but in only three studies, splicing experiments were developed to demonstrate the causal relationship between both $[(12,13)$ and the present study].

In several instances, it has been suggested that disruption of the pre-mRNA secondary structure might be at the basis of the aberrant splicing. In general, computer programs are used to predict the pre-mRNA mfe secondary structures $(18,22,32)$, some of which have been experimentally confirmed $(21,24)$. To test the hypothesis of pre-mRNA secondary structure disruption for exon 6 of the PDH E1 $\alpha$ gene, we decided to use the calculations of the RNAdraw program (30) taken by the RNA fold program from the "Vienna RNA package" [RNA fold (31)], a program that has been shown to generate welldetermined structures $(33,34)$. As a first approach, we decided to use only the $93 \mathrm{bp}$ of exon 6 of the gene in the mfe calculations. However, as exonic sequences can interact with intronic sequences in the pre-mRNA $(21,23)$ and these interactions might be indispensable for obtaining a well-stabilized structure, we decided to change the strategy by including sequences from the surrounding introns. Nevertheless, the mutations did, in both cases, disrupt the predicted secondary structure of the wild-type pre-mRNA sequence. These results urged us to further study the hypothesis by designing several new mutations that retain, disrupt, or restore the predicted wild-type mfe secondary structure $(690 \mathrm{C} \rightarrow \mathrm{A}, 666 \mathrm{~T} \rightarrow \mathrm{T}$, $671 \mathrm{~T} \rightarrow \mathrm{C}, 628 \mathrm{G} \rightarrow \mathrm{A}+708+118 \mathrm{~T} \rightarrow \mathrm{C})$. These mutations were created in the exon trap construct by site-directed mutagenesis and studied by exon trap analysis. However, no correlation could be found between the predicted structures and the splicing patterns.

A few reasons could be found to explain the lack of correlation between the predicted structures and the splicing patterns. First of all, the pre-mRNA secondary structure predicted by the RNAdraw program does not reflect the conformation of this RNA in vivo. Besides the fact that only part of the gene is taken into consideration for structure calculations, the computer program also does not take into account that these structures might be influenced by specific interactions of RNA-protein complexes such as the hnRNP A1 protein that might help to define the borders of mammalian introns $(35,36)$. A second possibility is that the predicted secondary structure exists but that it does not interfere in the splicing process. Indeed, Eperon et al. (37) have shown that the pre-mRNA secondary structure only sequesters splicing sites effectively when an alternative site is provided. When the alternative site is inactivated, the otherwise blocked site is used in vivo. Such sites might be revealed by an RNA duplex-unwinding activity, or by helix destabilization activity found in hnRNP preparations. In mammals, three putative helicases involved in mammalian pre-mRNA splicing have been identified, but their interactions with splicing sites have not yet been determined (38).

Nevertheless, we observed that the two mutations (660de$1 \mathrm{AA}$ and $661 \mathrm{~A} \rightarrow \mathrm{C}$ ) constructed in the purine-rich region of exon 6 were favoring the inclusion of this exon in the final
mRNA, when compared with the wild-type construct. Several studies have demonstrated that splicing of pre-mRNAs containing weak 3 ' splice site consensus sequences require splicing enhancer elements for activity (39-41). Nonsense mutations localized in ESEs are able to cause skipping of the respective exon, owing to a disruption of the interactions between an ESE and SR-rich proteins $(27,42)$. When comparing the purine-rich sequence of exon 6 (TGGAAAAGA) with ESE sequences published by Schaal and Maniatis (43), we observed that indeed the purine-rich sequence after mutation 660delAA (TGGAAGA) becomes more similar to a published ESE (YGGAGA, where $\mathrm{Y}$ is a pyrimidine), and the mutation $661 \mathrm{~A} \rightarrow \mathrm{C}$ produced a sequence that was shown to be part of the IgM M2 enhancer and three other ESEs identified by the SELEX technique (GGACAAGA - the underlined sequences are conserved and the three nucleotides in the middle are variable). It is possible that the mutation $660 \mathrm{~A} \rightarrow \mathrm{G}$ is localized in an important site of specific protein binding, leading to aberrant splicing. The new mutations produced in this region could make this site more favorable for being recognized by this or other proteins. Mutagenic studies in ESEs of the human $\beta$-globin gene support this theory. It was shown for this gene that a point mutation and a double mutation in an ESE did not affect the normal splicing of exon 2. Indeed, these mutations lead to increased binding of a new, unidentified $70-\mathrm{kD}$ protein. Interestingly, the authors found in another ESE that a point mutation, which does not change the amino acid sequence, had a dramatic effect on the mRNA splicing efficiency (27). Taking together the mutations of patient G.D., 660delAA, 661 A $\rightarrow$ C, $666 \mathrm{~T} \rightarrow \mathrm{C}$, and $671 \mathrm{~T} \rightarrow \mathrm{C}$, we can delimit a region of $12 \mathrm{bp}$ (between bp 42 and 56, Fig. 1) where the sequence changes are actively affecting splicing (inhibiting or enhancing the correct splicing of exon 6). This region could probably be a regulatory splicing region.

The in vivo splicing experiments in COS-7 cells result in either complete (mutation $628 \mathrm{G} \rightarrow \mathrm{A}$ ) or almost complete (mutation $660 \mathrm{~A} \rightarrow \mathrm{G}$ ) skipping of the PDH E1 $\alpha$ exon 6 for the mutant sequences, whereas approximately $50 \%$ skipping was observed for wild-type exon 6 . The appearance of a faint band containing exon 6 for mutant $660 \mathrm{~A} \rightarrow \mathrm{G}$ is in agreement with the in vivo situation in which only partial skipping of this exon is observed. The $50 \%$ skipping observed for the wild-type exon 6 sequence could possibly be explained on the basis that the weak 3 ' splice site of exon 6 requires an ESE to be recognized by the spliceosome complex. This splice site indeed shows the lowest value of the entire PDH E1 $\alpha$ gene when 3' and 5' splice site consensus values are calculated according to Shapiro and Senapathy (44). Absence or reduced production of a protein to interact with the ESE(s) in COS-7 cells could explain the partial skipping observed for this exon. The increased inclusion of exon 6 by the mutants could be explained by a better recognition of this protein or by the change of a site that is recognized by another protein that is present in these cells. Although specific SR-rich protein binding sites have been identified, individual SR-rich proteins are capable of recognizing a broad spectrum of weakly related sequences (27). 
In conclusion, we could demonstrate for the first time that exonic mutations lead to aberrant splicing in the PDH E1 $\alpha$ gene. We did not observe a relationship between disruption of the predicted secondary structure of the pre-mRNA and exon skipping. The mutagenic studies performed suggest the presence of a regulatory splicing region, most probably an ESE, localized in the middle region of exon 6 of the PDH E1 $\alpha$ gene. Further experiments should be performed to define this regulatory element and its respective protein interactions.

\section{REFERENCES}

1. Robinson BH 1995 Lactic acidemia (disorders of pyruvate carboxylase, pyruvate dehydrogenase). In: Scriver CR, Beaudet AL, Sly WS, Valle D (eds) The Metabolic and Molecular Bases of Inherited Disease. McGraw-Hill, New York, pp 1479-1499

2. Brown GK, Otero LJ, LeGris M, Brown RM 1994 Pyruvate dehydrogenase deficiency. J Med Genet 31:875-879

3. Dahl H-HM, Brown GK, Brown RM, Hansen LL, Kerr DS, Wexler ID, Patel MS, De Meirleir L, Lissens W, Chun K, MacKay N, Robinson BH 1992 Mutations and polymorphisms in the pyruvate dehydrogenase E1 $\alpha$ gene. Hum Mutat 1:97-102

4. Lissens W, De Meirleir L, Seneca S, Benelli C, Marsac C, Poll-The BT, Briones P, Ruitenbeek W, van Diggelen O, Chaigne D, Ramaekers V, Liebaers I 1996 Mutation analysis of the pyruvate dehydrogenase $\mathrm{E} 1 \alpha$ gene in eight patients with a pyruvate dehydrogenase complex deficiency. Hum Mutat 7:46-51

5. De Meirleir L, Specola N, Seneca S, Lissens W 1998 Pyruvate dehydrogenase E1 $\alpha$ deficiency in a family: different clinical presentation in two sibilings. J Inherit Metab Dis 21:224-226

6. Kerr DS, Wexler ID, Tripatara A, Patel MS 1996 Human defects of the pyruvate dehydrogenase complex. In: Patel MS, Roche TE, Harris RA (eds) Alpha-keto Acid Dehydrogenase Complexes. Birkhauser Verlag, Basel, pp 249-269

7. Lissens W, De Meirleir L, Seneca S, Liebaers I, Brown GK, Brown RM, Ito M, Naito E, Kuroda Y, Kerr DS, Wexler ID, Patel MS, Robinson BH, Seyda A 2000 Mutations in the X-linked pyruvate dehydrogenase (E1) $\alpha$ subunit gene $(P D H A 1)$ in patients with a pyruvate dehydrogenase complex deficiency. Hum Mutat 15:209-219

8. De Meirleir L, Lissens W, Benelli C, Ponsot G, Desguerre I, Marsac C, Rodriguez D, Saudubray J-M, Poggi F, Liebaers I 1994 Aberrant splicing of exon 6 in the pyruvate dehydrogenase-E1 $\alpha$ mRNA linked to a silent mutation in a large family with Leigh's encephalomyelopathy. Pediatr Res 36:707-712

9. Chun K, MacKay N, Petrova-Benedict R, Federico A, Fois A, Cole DEC, Roberteson E, Robinson BH 1995 Mutations in the X-linked E1 $\alpha$ subunit of pyruvate dehydrogenase: exon skipping, insertion of duplicate sequence, and missense mutation leading to the deficiency of the pyruvate dehydrogenase complex. Am J Hum Genet $56: 558-569$

10. Krawczak M, Reiss J, Cooper DN 1992 The mutational spectrum of single base pair substitutions in mRNA splice junctions of human genes: causes and consequences. Hum Genet 90:41-54

11. Steingrimsdottir H, Rowley G, Dorado G, Cole J, Lehmann AR 2081992 Mutations which alter splicing in the human hypoxanthine-guanine phosphoribosyltransferase gene. Nucleic Acids Res 20:1201-1208

12. Fukao T, Yamaguchi S, Wakazono A, Orii T, Hoganson G, Hashimoto T 1994 Identification of a novel exonic mutation at -13 from 5 ' splice site causing exon skipping in a girl with mitochondrial acetoacetyl-coenzyme A thiolase deficiency. J Clin Invest 93:1035-1041

13. Liu W, Qian C, Francke U 1997 Silent mutation induces exon skipping of fibrillin-1 gene in Marfan syndrome. Nat Genet 16:328-329

14. Pie J, Casals N, Casale CH, Buesa C, Mascaro C, Barcelo A, Rolland MO, Zabot T, Haro D, Eyskens F, Divry P, Hegardt FG 1997 A nonsense mutation in the 3-hydroxy-3-methylglutaryl-CoA lyase gene produces exon skipping in two patients of different origin with 3-hydroxy-3-methylglutaryl-CoA lyase deficiency. Biochem J 323:329-335

15. Babcock D, Gasner C, Francke U, Malsen C 1998 A single mutation that results in an Asp to His substitution and partial exon skipping in a family with congenital contractual arachnodactyly. Hum Genet 103:22-28

16. Sakuntabhai A, Hammami-Hauasli N, Bodemer C, Rochat A, Prost C, Barrandon Y, de Prost Y, Lathrop M, Wojnarowska F, Bruckner-Tunderman L, Hovnanian A 1998 Deletions within COL7A1 exons distant from consensus splice sites alter splicing and produce shortened polypeptides in dominant dystrophic epidermolysis bullosa. Am J Hum Genet 63:737-748

17. Mendez M, Sorkin L, Rossetti MV, Astrin KH, del C Batlle AM, Parera VE, Aizencang G, Desnick RJ 1998 Familial porphyria cutanea tarda: characterization of seven novel uroporphyrinogen decarboxylase mutations and frequency of common hemochromatosis alleles. Am J Hum Genet 63:1363-1375

18. Hoffmeyer S, Nürnberg P, Ritter H, Fahsold R, Leistner W, Kaufmann D, Krone W 1998 Nearby stop codons in exons of the neurofibromatosis type 1 gene are disparate splice effectors. Am J Hum Genet 62:269-277

19. Kanai N, Yanai F, Hirose S, Nibu K, Izuhara K, Tani T, Kubota T, Mitsudome A 1999 A G to A transition at the last nucleotide of exon 6 of the $\gamma \mathrm{c}$ gene $(868 \mathrm{G} \rightarrow \mathrm{A})$ may result in either a splice or missense mutation in patients with $\mathrm{X}$-linked severe combined immunodeficiency. Hum Genet 104:36-42

20. Lorson CL, Androphy EJ 2000 An exonic enhancer is required for inclusion of an essential exon in the SMA-determining gene SMN. Hum Mol Genet 9:259-265

21. Clouet d'Orval BC, d'Aubenton Carafa YA, Sirand-Pungnet P, Gallego M, Brody E, Marie J 1991 RNA secondary structure repression of a muscle-specific exon in HeLa cell nuclear extracts. Science 252:1823-1828

22. Libri D, Piseri A, Fiszman MY 1991 Tissue-specific splicing in vivo of the $\beta$-tropomyosin gene: dependence on an RNA secondary structure. Science 252:1842-1845

23. Coleman TP, Roesser JR 1998 RNA secondary structure: an important cis-element in rat calcitonin/CGRP pre-messenger RNA splicing. Biochemistry 37:15941-15950

24. Muro AF, Caputi M, Pariyarath R, Pagani F, Buratti E, Baralle FE 1999 Regulation of fibronectin EDA exon alternative splicing: possible role of RNA secondary structure for enhancer display. Mol Cell Biol 19:2657-2671

25. Cooper TA, Mattox W 1997 The regulation of splice site selection, and its role in human disease. Am J Hum Genet 61:259-266

26. Coulter LR, Landree MA, Cooper TA 1997 Identification of a new class of exonic splicing enhancers by in vivo selection. Mol Cell Biol 17:2143-2150

27. Schaal TD, Maniatis T 1999 Multiple distinct splicing enhancers in the protein-coding sequences of a constitutively spliced pre-mRNA. Mol Cell Biol 19:261-273

28. Koike K, Urata Y, Matsuo S, Koike M 1990 Characterization and nucleotide sequence of the gene encoding the human pyruvate dehydrogenase $\alpha$ subunit. Gene 93:307-311

29. Hanahan D 1983 Studies on transformation of E. coli with plasmid. J Mol Biol $166: 557-580$

30. Matzura O, Wennborg A 1996 RNAdraw: an integrated program for RNA secondary structure calculation and analysis under 32-bit Microsoft Windows. Comput Appl Biosci 12:247-249

31. Hofacker IL, Fontana W, Stadler PF, Bonhoeffer LS, Tacker M, Schuster P 1994 Fast folding and comparison of RNA secondary structures. Monatsh Chem 125:167-188

32. Staffa A, Acheson NH, Cochrane A 1997 Novel exonic elements that modulate splicing of the human fibronectin EDA exon. J Biol Chem 272:33394-33401

33. Huynen MA, Perelson A, Vieira WA, Stadler PF 1996 Base pairing probabilities in a complete HIV-1 RNA. J Comput Biol 2:253-274

34. Huynen M, Gutell RR, Konings DA 1997 Assessing the reliability of RNA fold using statistical mechanisms. J Mol Biol 267:1104-1112

35. Solnick D, Lee SI 1987 Amount of RNA secondary structure required to induce an alternative splice. Mol Cell Biol 7:3194-3198

36. Blanchete M, Chabot B 1999 Modulation of exon skipping by high-affinity hnRNP A1-binding sites and by intron elements that repress splice site utilization. EMBO J 18:1939-1952

37. Eperon LP, Graham IR, Griffiths AD, Eperon IC 1988 Effects of RNA secondary structure on alternative splicing of pre-mRNA: is folding limited to a region behind the transcribing RNA polymerase? Cell 54:393-401

38. Will CL, Lührmann R 1997 Protein functions in pre-mRNA splicing. Curr Opin Cell Biol 9:320-328

39. Lopez AJ 1998 Alternative splicing of pre-mRNA: developmental consequences and mechanisms of regulation. Annu Rev Genet 32:279-305

40. Dye BT, Buvoli M, Mayer SA, Lin C-H, Patton JG 1998 Enhancer elements activate the weak 3' splice site of $\alpha$-tropomyosin exon 2. RNA 4:1523-1536

41. Yue B-G, Akusjärvi G 1999 A downstream splicing enhancer is essencial for in vitro pre-mRNA splicing. FEBS Lett 451:10-14

42. Watakabe A, Tanaka K, Shimura Y 1993 The role of exon sequences in splice site selection. Genes Dev 7:407-418

43. Schaal TD, Maniatis T 1999 Selection and characterization of pre-mRNA splicing enhancers: identification of novel SR protein-specific enhancer sequences. Mol Cell Biol 19:1705-1719

44. Shapiro MB, Senapathy P 1987 RNA splice junctions of different classes of eukaryotes: sequence statistics and functional implications in gene expression. Nucleic Acids Res 15:7155-7174 